\title{
Total horizontal derivatives of potential field three- dimensional structure tensor and their application to detect source edges
}

\author{
Shuai Zhou' ${ }^{1} \cdot$ Danian Huang $^{1} \cdot$ Jian Jiao $^{1}$
}

Received: 9 November 2015/Accepted: 4 April 2016/Published online: 12 April 2016

(C) Akadémiai Kiadó 2016

\begin{abstract}
Edge detection of the geological structures horizontal position is the vital task of the potential field interpretation, and some traditional edge detectors have poor ability in delineating edges clearly and accurately. Some people made efforts to purpose new methods which can balance the weak and strong anomalies simultaneously. In this paper, we purposed new edge detection methods based on directional total horizontal derivatives of three-dimensional structure tensor for the causative sources. In order to balance weak and strong amplitude anomalies simultaneously, we used the vertical derivatives of potential field to normalize the new defined filters. For the complex geological situation with positive and negative gravity or magnetic anomalies, we introduced a new strategy to reduce the false edges. The presented filters were tested on synthetic and real potential field data to verify its feasibility. All of the results have shown the new edge detection methods can not only display the sources edges precisely and clearly, but also bring out more geological subtle details.
\end{abstract}

Keywords Total horizontal derivative $\cdot$ Edge detection $\cdot$ Structure tensor $\cdot$ Potential field

\section{Introduction}

There are many edge detection filters used to detect geological structure edges in the process of potential field data interpretation. Cordell (1979), Cordell and Grauch (1985) indicated that the maximum values of the total horizontal derivative correspond to the

Jian Jiao

626629279@qq.com

Shuai Zhou

zhoushuai14@mails.jlu.edu.cn

Danian Huang

dnhuang@jlu.edu.cn

1 College of Geoexploration Science and Technology, Jilin University, Changchun 130026, China 
sources edges. Hood and Teskey (1989) used the zero contours of vertical derivative to delineate edges of gravity and magnetic field. Nabighian $(1972,1984)$ had delineated the geological bodies edges using the amplitude of the analytical signal. But these methods have no ability in displaying weak and strong amplitude anomalies simultaneously. Miller and Singh (1994) proved the ratio of the total horizontal derivative and vertical derivative, called Tilt angle method, can balance different amplitude anomalies. Verduzco et al. (2004) calculated the total horizontal derivative of the tilt angle to increase the edge detecting resolution. Wijns et al. (2005) have developed a new balanced filter theta map for edge detecting, which used the ratio of the analytic signal amplitude and the total horizontal derivative. Cooper and Cowan (2008) used the balanced windowed standard deviation to enhance the edges, which can balance the different amplitude anomalies. Zhou et al. (2013) developed new edge detector based on the eigenvalues of gravity tensor data for the anomalies with positive and negative density contrast edge detecting.

Structure tensor is a traditional partial structure analysis tool, which is widely used in image processing, segmentation and feature detection. Weickert (1999a, b) presented that the structure tensor can be used to delineate the image edges and corners. Three-dimensional structure tensor can be effective in representation of the local motion information of video object, and has been exploited for performing video object segmentation (Wang and Ma 2003). Jeong et al. (2006) used the structure tensor to the seismic images faults recognition for the geophysical application. Sertcelik and Kafadar (2012) introduced the two-dimensional structure tensor with Gaussian function convolution for the potential field edge detecting, but the method failed to outline the deep and shallow anomalies simultaneously. Then Ma and Huang (2015) presented a new method normalizing the defined edge detector, which used the ratio of the vertical derivative and total horizontal derivatives of the 2D structure tensor eigenvalue. Yuan et al. (2014) pointed the Gaussian envelop will smooth the potential field data, and redefined the structure tensor eigenvalue without the Gaussian envelop to make the detected edges more clearly.

In this paper, we defined the directional total horizontal derivatives (THD) of 3D structure tensor. And the new edge detectors were purposed with better resolution compared with the traditional methods. We applied the new filters to the synthetic complex model data and real measured gravity and magnetic data to show the performance of the new edge detectors.

\section{Directional THD of 3D structure tensor}

Sertcelik and Kafadar (2012) defined two-dimensional structure tensor matrix with Gaussian envelop, which forms as the following equation:

$$
T_{\sigma}=E_{\sigma} * T=\left[\begin{array}{ll}
E_{\sigma} *\left(\frac{\partial \mathrm{G}_{\mathrm{z}}}{\partial x}\right)^{2} & E_{\sigma} *\left(\frac{\partial \mathrm{G}_{\mathrm{z}}}{\partial x} \frac{\partial \mathrm{G}_{\mathrm{z}}}{\partial y}\right) \\
E_{\sigma} *\left(\frac{\partial \mathrm{G}_{\mathrm{z}}}{\partial y} \frac{\partial \mathrm{G}_{\mathrm{z}}}{\partial x}\right) & E_{\sigma} *\left(\frac{\partial \mathrm{G}_{\mathrm{z}}}{\partial y}\right)^{2}
\end{array}\right]=\left[\begin{array}{ll}
T_{11} & T_{12} \\
T_{21} & T_{22}
\end{array}\right],
$$

where $E_{\sigma}$ is the function of Gaussian envelop, $G$ is the potential, $G_{z}$ represents the original gravity or magnetic anomaly, $\frac{\partial G_{z}}{\partial x}$ and $\frac{\partial G_{z}}{\partial y}$ are the derivatives of $G_{z}$ in $x$ and $y$ directions, the symbol ' $*$ ' is used for convolution. The eigenvalues of the above $2 \mathrm{D}$ structure tensor have 
the ability in detecting the sources edges and corners. Yuan et al. (2014) redefined the 2D structure tensor without the Gaussian envelop and presented new balanced edge detectors to enhance the sources edges. The 2D structure tensor without Gaussian envelop is defined as:

$$
T=\left[\begin{array}{ll}
\left(\frac{\partial G_{z}}{\partial x}\right)^{2} & \frac{\partial G_{z}}{\partial x} \frac{\partial G_{z}}{\partial y} \\
\frac{\partial G_{z}}{\partial y} \frac{\partial G_{z}}{\partial x} & \left(\frac{\partial G_{z}}{\partial y}\right)^{2}
\end{array}\right]=\left[\begin{array}{ll}
T_{11} & T_{12} \\
T_{21} & T_{22}
\end{array}\right] .
$$

We give the 3D structure tensor of potential field without Gaussian envelop, and develop new edge detectors based on it. The expression of 3D structure tensor is:

$$
T=\left[\begin{array}{lll}
\left(\frac{\partial G_{z}}{\partial x}\right)^{2} & \frac{\partial G_{z}}{\partial x} \frac{\partial G_{z}}{\partial y} & \frac{\partial G_{z}}{\partial x} \frac{\partial G_{z}}{\partial z} \\
\frac{\partial G_{z}}{\partial y} \frac{\partial G_{z}}{\partial x} & \left(\frac{\partial G_{z}}{\partial y}\right)^{2} & \frac{\partial G_{z}}{\partial y} \frac{\partial G_{z}}{\partial z} \\
\frac{\partial G_{z}}{\partial z} \frac{\partial G_{z}}{\partial x} & \frac{\partial G_{z}}{\partial z} \frac{\partial G_{z}}{\partial y} & \left(\frac{\partial G_{z}}{\partial z}\right)^{2}
\end{array}\right]=\left[\begin{array}{lll}
T_{11} & T_{12} & T_{13} \\
T_{21} & T_{22} & T_{23} \\
T_{31} & T_{32} & T_{33}
\end{array}\right] .
$$

Beiki (2010) analyzed the analytic signals of the potential field gradient tensor, and defined analytic signals, called directional analytic signals for every row of the potential field gradient tensor matrix. The directional analytic signals can be written as:

$$
\begin{aligned}
& A_{x}=\sqrt{G_{x x}^{2}+G_{x y}^{2}+G_{x z}^{2}}, \\
& A_{y}=\sqrt{G_{y x}^{2}+G_{y y}^{2}+G_{y z}^{2}}, \\
& A_{z}=\sqrt{G_{x z}^{2}+G_{y z}^{2}+G_{z z}^{2}},
\end{aligned}
$$

where, $G$ is the gravity or magnetic potential, $A_{x}$ and $A_{y}$ can outline the N-S and E-W edges, $A_{z}$ is the traditional analytic signal. Beiki (2010) pointed that the directional analytic signals $A_{x}$ and $A_{y}$ can be used for edge detecting, and the edge detector $E D$ based on directional analytical signals is:

$$
E D=\sqrt{\left(A_{x}\right)^{2}+\left(A_{\mathrm{y}}\right)^{2}} .
$$

Yuan and Geng (2014) defined the directional total horizontal derivatives of gravity gradient tensor, but the defined filter has no ability in balancing deep and shallow sources anomalies simultaneously. Marson and Klingele (2015) had pointed that the total horizontal derivatives has better resolution than analytic signal in edge detection. Similar to the definition of directional analytic signals and total horizontal derivatives of potential field gradient tensor in $x$ and $y$ directions, we define the directional total horizontal derivatives of potential field 3D structure tensor [Eq. (3)] in $x$ and $y$ directions as:

$$
T H D_{x}=\sqrt{\left(T_{12}\right)^{2}+\left(T_{13}\right)^{2}},
$$




$$
T H D_{\mathrm{y}}=\sqrt{\left(T_{21}\right)^{2}+\left(T_{23}\right)^{2}}
$$

where, the maximum values of $T H D_{x}$ and $T H D_{y}$ indicate the $\mathrm{N}-\mathrm{S}$ and $\mathrm{E}-\mathrm{W}$ edges, respectively. The new edge detector with a better resolution based on the $x$ and $y$ directional horizontal derivatives can be expressed as:

$$
T H D T=\sqrt{\left(T H D_{x}\right)^{2}+\left(T H D_{\mathrm{y}}\right)^{2}} .
$$

Hsu et al. (1996) used the high-order derivative of analytical signal to obtain clear and accurate edges position. In order to further increase the edge detecting resolution, we use the high-order vertical derivative of original potential field to define a new detector. Highorder vertical derivative calculation will increase the noise effect, so we just compute the first-order vertical derivative of the original potential field to obtain more accurate edges position. The first-order directional total horizontal derivatives are expressed as:

$$
\begin{aligned}
\text { FTHD }_{x} & =\sqrt{\left[\frac{\partial}{\partial x}\left(\frac{\partial G_{z}}{\partial z}\right) \cdot \frac{\partial}{\partial y}\left(\frac{\partial G_{z}}{\partial z}\right)\right]^{2}+\left[\frac{\partial}{\partial x}\left(\frac{\partial G_{z}}{\partial z}\right) \cdot \frac{\partial}{\partial z}\left(\frac{\partial G_{z}}{\partial z}\right)\right]^{2}}, \\
\text { FTHD }_{y} & =\sqrt{\left[\frac{\partial}{\partial y}\left(\frac{\partial G_{z}}{\partial z}\right) \cdot \frac{\partial}{\partial x}\left(\frac{\partial G_{z}}{\partial z}\right)\right]^{2}+\left[\frac{\partial}{\partial y}\left(\frac{\partial G_{z}}{\partial z}\right) \cdot \frac{\partial}{\partial z}\left(\frac{\partial G_{z}}{\partial z}\right)\right]^{2}} .
\end{aligned}
$$

The related new edge detector is:

$$
F T H D T=\sqrt{\left(F T H D_{x}\right)^{2}+\left(F T H D_{\mathrm{y}}\right)^{2}} .
$$

The maximum values of the new purposed edge detectors THDT and FTHDT delineate the sources edges, but fail to display the edges caused by small and large amplitude sources simultaneously. The first-order vertical derivative of original potential field data is used to normalize the new detectors. The normalized THDT detector is defined as:

$$
N T=\tan ^{-1}\left[\frac{T H D T}{\left|\frac{\partial G_{z}}{\partial z}\right|^{2}+k \cdot \max (T H D T)}\right],
$$

where $k$ is a constant value between 0 and 0.1 . The introduction of $k$ can reduce the false edges in the geological situation contained positive and negative potential field anomalies. The small value of $k$ will increase the balanced ability of the edge detector, and the large value of $k$ makes the small amplitude anomalies edges blurred. For the situation only contained positive or negative anomalies, $k$ should be set as zero. And in other situation, the interpreter should decide $k$ value from the result.

We also use the first-order vertical derivative of the original potential field data to balance the new edge detector FTHDT to get more accurate and clear edges position. The new normalized edge detector is expressed as:

$$
N F T=\tan ^{-1}\left[\frac{F T H D T \cdot t}{\left|G_{z z}\right|^{2}+k \cdot \max (F T H D T \cdot t)}\right],
$$


and,

$$
t=\left(\frac{\min \left(\mathrm{G}_{\mathrm{zz}}\right)}{\max \left(G_{z z z}\right)}\right)^{2},
$$

where, $t$ is a constant value, to make Eq. (15) has mathematical significance, and min and max represent the global minimum and maximum value of the related potential field. And $G_{z z}, G_{z z z}$ are the different-order vertical derivative of potential $G$ in $z$ direction.

The different-order horizontal derivatives of gravity or magnetic potential $G$ can be calculated in space domain. And we compute potential field $G$ from the original potential field data $G_{z}$ in frequency domain, the program used in Cooper (2009) is applied for the components calculation, exactly the ' $\mathrm{dz}$ ' calculation subroutine is modified for frequency domain calculation. When we calculate the edge detectors, we need to calculate the vertical derivatives of $G_{x y}, G_{x z}, G_{y z}$ and $G_{z z}$. Calculating vertical derivative by the use of the vertical-derivative operator will amplify the noise effect. We can substitute the vertical derivatives with the horizontal derivatives of the gravity gradient tensor calculated in space domain, which will not increase the noise effect so intensively.

$$
\begin{aligned}
& \frac{\partial G_{x y}}{\partial z}=\frac{\partial G_{z x}}{\partial y}, \\
& \frac{\partial G_{x z}}{\partial z}=\frac{\partial G_{z z}}{\partial x}, \\
& \frac{\partial G_{y z}}{\partial z}=\frac{\partial G_{z z}}{\partial y},
\end{aligned}
$$

where, $G$ denotes potential field. For the calculation of the vertical derivative of $G_{z z}$, we can use the following Laplace equation (Blakely 1995) to compute $G_{z z z}$ :

$$
G_{z z z}=\frac{\partial^{2} G_{z}}{\partial z^{2}}=-\left(\frac{\partial^{2} G_{z}}{\partial x^{2}}+\frac{\partial^{2} G_{z}}{\partial y^{2}}\right)=-\left(\frac{\partial G_{x z}}{\partial x}+\frac{\partial G_{y z}}{\partial y}\right) .
$$

\section{Application to synthetic model data}

In order to explain edge detecting effectiveness of the directional total horizontal derivatives of 3D structure tensor in $x$ and $y$ directions, we compare $T H D_{x}$ and $T H D_{y}$ with the directional analytical signals.

Figure 1a shows the gravity field data generated by the prism with depth $1 \mathrm{~km}$, thickness $0.5 \mathrm{~km}$ and positive density contrast $0.1 \mathrm{~g} / \mathrm{cm}^{3}$. The rectangle in Fig. 1a shows the horizontal position of the prism. Figure $1 \mathrm{~b}-\mathrm{d}$ show that the maximum values of $A_{x}, A_{y}$ and $E D$ indicate the sources edges. $T H D_{x}, T H D_{y}$ and THDT have better resolution than $A_{x}, A_{y}$ and $E D$, which are showed in Fig. 1-g. Compared the detector edge detecting results based on directional analytic signals (Beiki 2010) with the new defined edge detector THDT, THDT can delineate the edges more precisely. The maximum values of $F T H D_{x}$, $F T H D_{y}$ and FTHDT can make the prism edges more clearly and accurately (Fig. $1 \mathrm{~h}-\mathrm{j}$ ). 
(a)

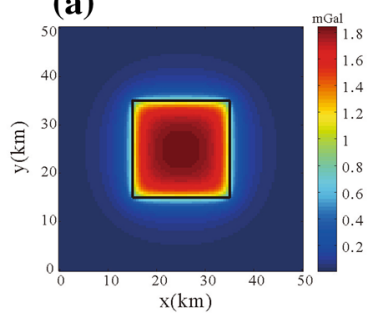

(b)

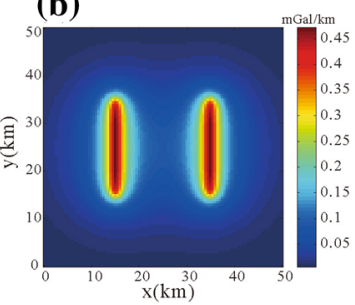

(e)

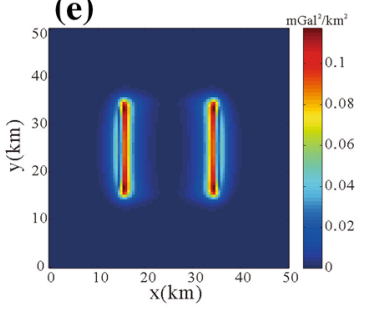

(h)

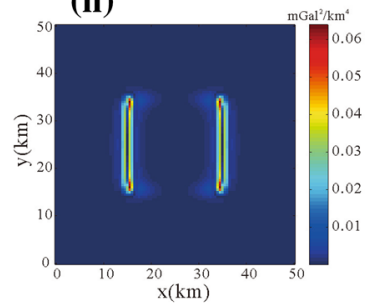

(c)

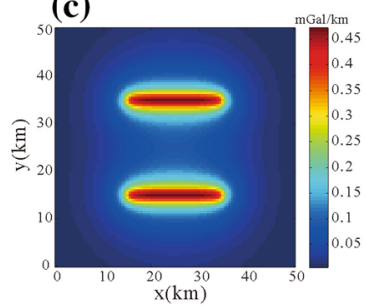

(f)

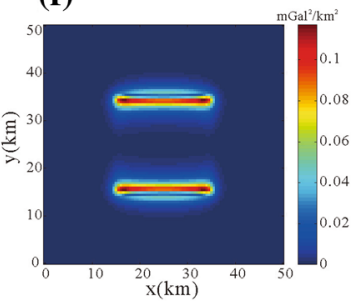

(i)

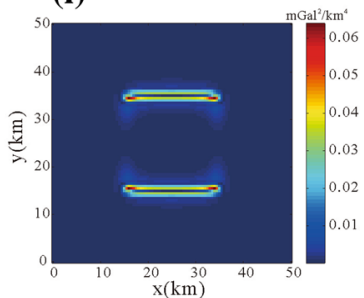

(d)

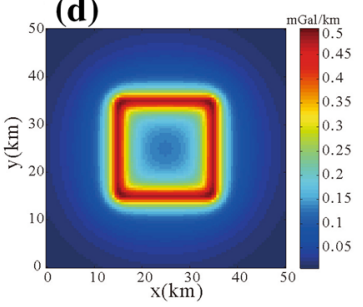

(g)

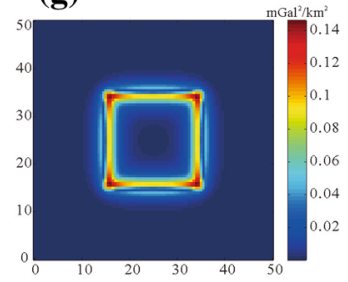

(j)

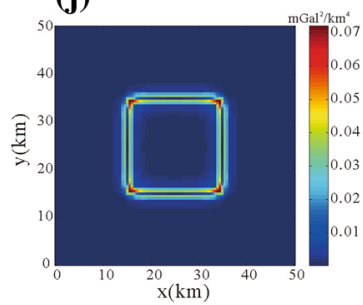

Fig. 1 Comparison between directional analytical signals and our method. a The synthetic gravity anomaly caused by a prism. b $A_{x}$ of the data. $\mathbf{c} A_{y}$ of the data. d $E D$ of the data. e $T H D_{x}$ of the data. $\mathbf{f} T H D_{y}$ of the data. g THDT of the data. h $F T H D_{x}$ of the data. i $F T H D_{y}$ of the data. j FTHDT of the data

We construct a new model 1 with four prisms, and the depth of number 1 and 3 prisms is $1.5 \mathrm{~km}$, the depth of number 2 and 4 prisms is $1 \mathrm{~km}$ (Fig. 2). The density contrast and thickness of four prisms is same with $0.1 \mathrm{~g} / \mathrm{cm}^{3}$ and $0.5 \mathrm{~km}$, respectively. The corresponding gravity anomaly is showed in Fig. 3.

In order to test the performance of the proposed edge detectors, we chose the traditional detectors TA_THDR (total horizontal derivative of the tilt angle) and Theta map for comparison. Miller and Singh (1994) proposed edge detector Tilt angle (TA) to balance different amplitude anomalies, and it can be expressed as: 

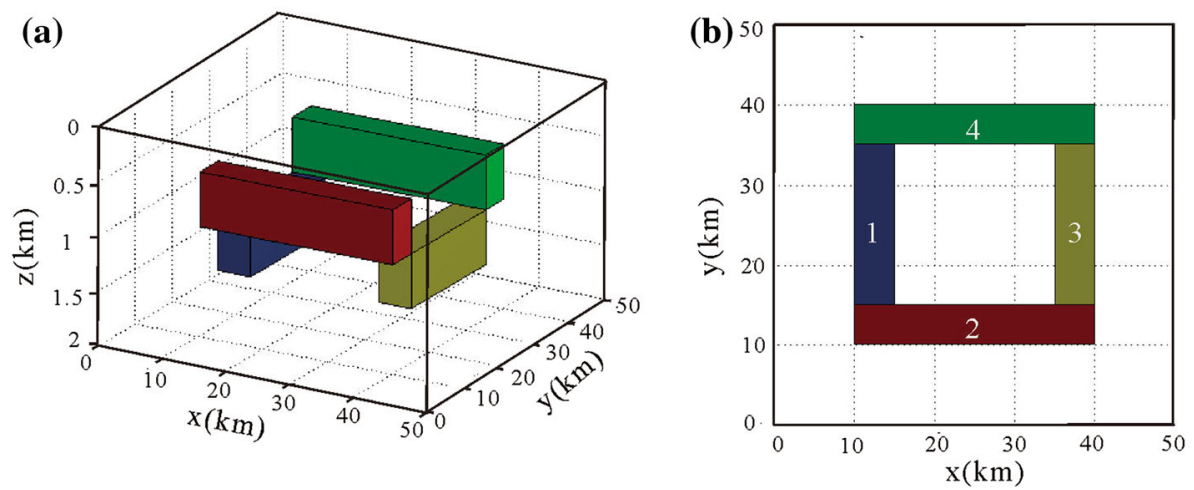

Fig. 2 Synthetic model 1. a 3D view of the model. b Plan view of the synthetic model

Fig. 3 The gravity anomaly caused by model 1 with positive density contrast

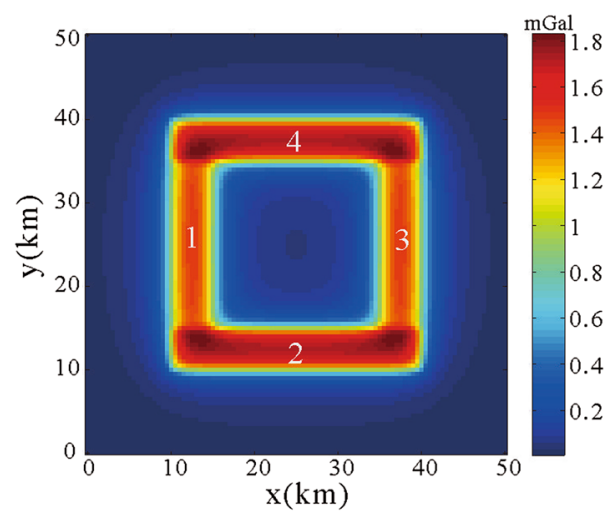

$$
T A=\tan ^{-1}\left(\frac{G_{z z}}{\sqrt{\left(G_{z x}\right)^{2}+\left(G_{z y}\right)^{2}}}\right),
$$

where $G$ denotes the potential field. Verduzco et al. (2004) suggested using the total horizontal derivative of the tilt angle (TA_THDR) for edge detecting, which can be given by:

$$
T A \_T H D R=\sqrt{\left(\frac{\partial T A}{\partial x}\right)^{2}+\left(\frac{\partial T A}{\partial y}\right)^{2}} .
$$

Wijns et al. (2005) used the analytic signal amplitude to normalize the total horizontal derivative of the potential field, and developed a new balanced filter theta map. The expression can be given by:

$$
\text { Theta }=\frac{\sqrt{\left(G_{z x}\right)^{2}+\left(G_{z y}\right)^{2}}}{\sqrt{\left(G_{z x}\right)^{2}+\left(G_{z y}\right)^{2}+\left(G_{z z}\right)^{2}}} .
$$


(a)

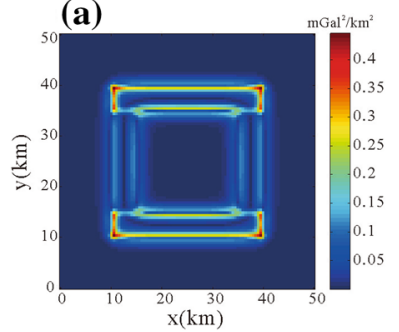

(d)

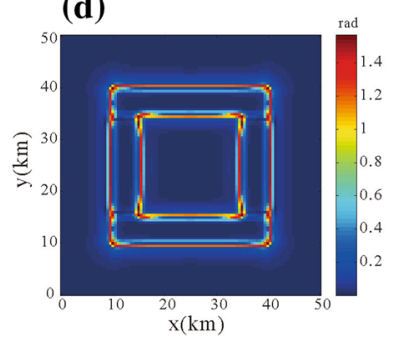

(b)

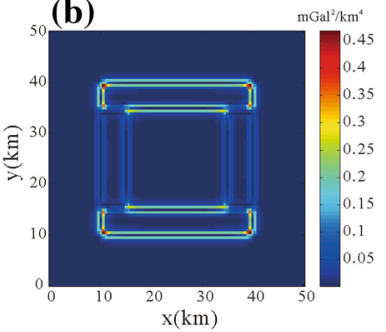

(e)

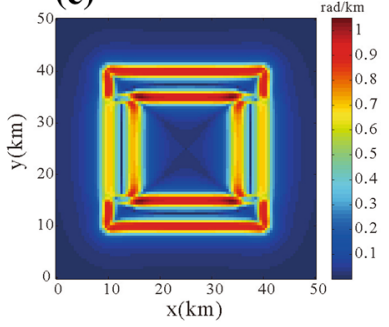

(c)

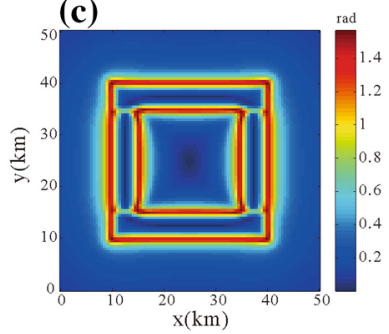

(f)

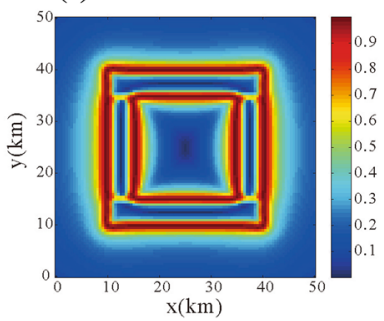

Fig. 4 Edges detected by different methods of model 1. a THDT of the data. b FTHDT of the data. c NT of the data. d NFT of the data. e TA_THDR of the data. f Theta map of the data

The maximum values of the TA_THDR and Theta map indicate the edges.

Figure 4 shows the edge detecting results of different method. THDT and FTHDT have no ability in outlining the deep prisms edges clearly (number 1 and 3 prisms), which are showed in Fig. 4a and b. The normalized new edge detectors NT and NFT, showed in Fig. $4 \mathrm{c}$ and $\mathrm{d}$, can overcome the problem and have a better performance in balancing small and large amplitude anomalies simultaneously, compared with the traditional filters TA_THDR and Theta map (Fig. 4e, f). When using Eq. (14) and (15) for NT and NFT calculation, the parameter $\mathrm{k}$ is selected as 0 for this situation with only positive gravity anomaly.

In order to further test the performance of the new edge detectors, we construct a new model 2 similar to the model 1, but with the contrasted density of number 1 and 4 prisms is $0.1 \mathrm{~g} / \mathrm{cm}^{3}$, the other is $-0.1 \mathrm{~g} / \mathrm{cm}^{3}$. Figure 5 shows the caused gravity anomaly of new model 2, and Fig. 6 shows the different method edge detecting results. In this situation, our new purposed methods can extract the prisms edges clearly without other additional edges. But the traditional method TA_THDR and Theta map methods will bring false edges, which will lead to wrong interpretation results. Here, $k$ is chosen as 0.02 for the new edge detectors $N T$ and NFT calculation.

To further test the stability of the new detectors, we add $2 \%$ Gaussian noise to the gravity anomaly caused by model 2 in Fig. 5. The value of $k$ is set as 0.02 for the new edge detector NT and NFT calculation. Due to the noise effect, detectors TA_THDR and Theta map fail to display the edges and they have poor performance (Fig. 7e, f). The noise has little influence on the new detectors THDT and FTHDT, showed in Fig. 7a and b, but the two filters can't balance the deep and shallow anomalies. The edges detected by $N T$ and $N F T$ can outline the edges effectively without any other false edges. Compared Fig. $7 \mathrm{~d}$ with (c), we can see that NFT has better resolution than NT. Depending on the Eq. (14) and (15), we can see that the detecting edges by the $N T$ and $N F T$ filters are related to the $\mathrm{k}$ parameter. Then we test the application effect of the different values of $\mathrm{k}$ for this synthetic 
Fig. 5 The gravity anomaly caused by model 2 with positive and negative density contrast

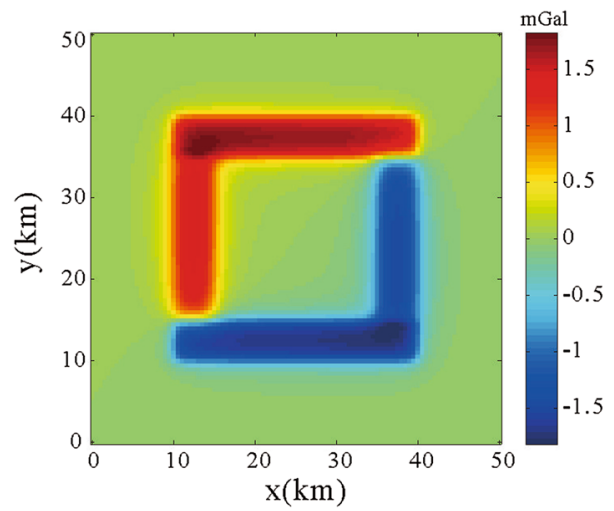

(a)

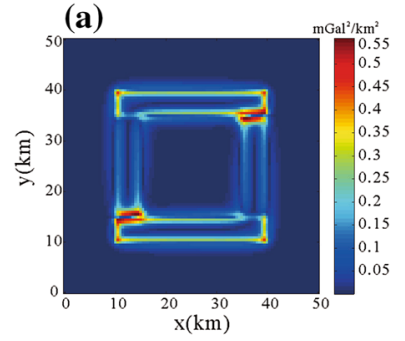

(d)

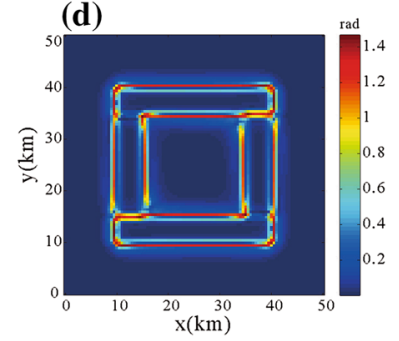

(b)

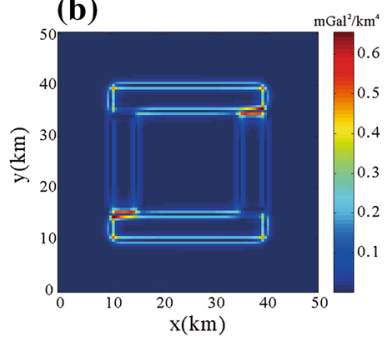

(e)

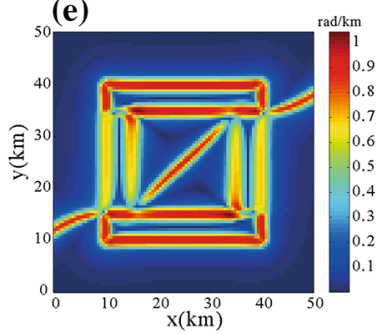

(c)

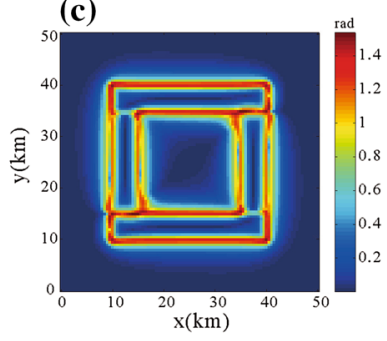

(f)

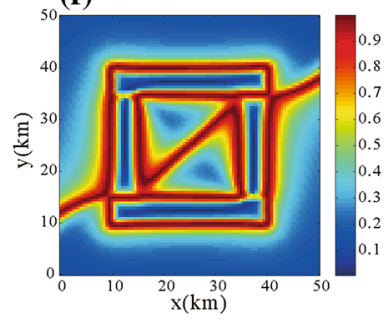

Fig. 6 Edges detected by different methods of model 2. a THDT of the data. b FTHDT of the data. c NT of the data. d NFT of the data. e TA_THDR of the data. f Theta map of the data

gravity anomaly. The factor $k$ values are selected as $0.2,0.02$ and 0.002 for comparison, and Fig. 8 shows the new detectors NT and NFT edge detecting results. The edges detected by the filters with the selected $\mathrm{k}$ value 0.02 obtain satisfactory result (Fig. 8b, e). The large value of $k$ equaling to 0.2 reduces the effectiveness of balancing different-amplitude anomalies. The detected edges of deep prisms 1 and 3 are not so clearly compared with the other shallow prisms (Fig. 8a, d). Figure 8c and f show the recognized edges result with small $k$ value 0.002 with a better balanced ability, but the detectors can't reduce the false edges caused by positive and negative anomalies effectively and are more affected by the noise. We suggest that the interpreter should use different values of $k$ to calculate the new normalized filters and choose the reasonable result. 


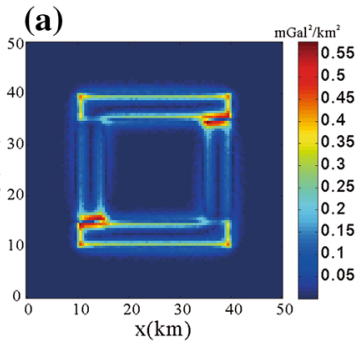

(d)

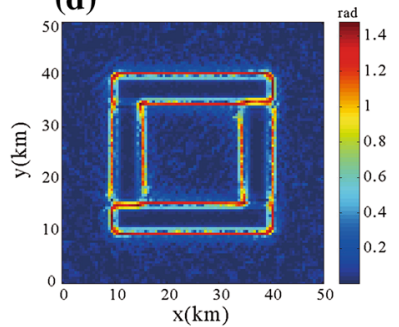

(b)

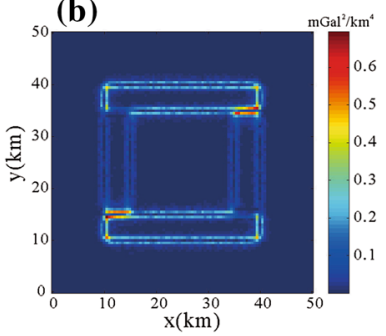

(e)

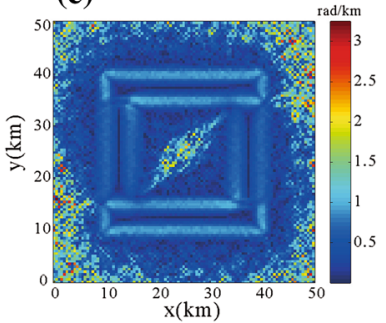

(c)

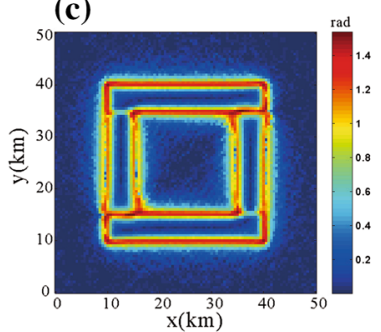

(f)

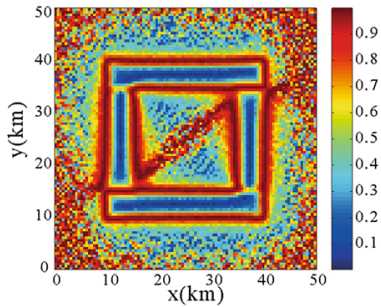

Fig. 7 Edges detected by different methods of model 2 added with $2 \%$ Gaussian noise. a THDT of the data. b FTHDT of the data. $\mathbf{c}$ NT of the data. d NFT of the data. e TA_THDR of the data. $\mathbf{f}$ Theta map of the data

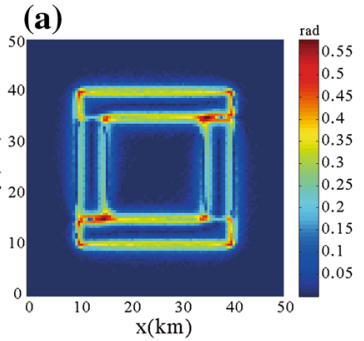

(d)

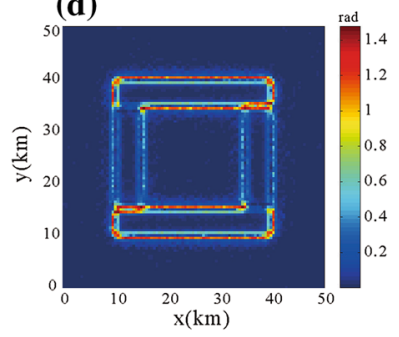

(b)

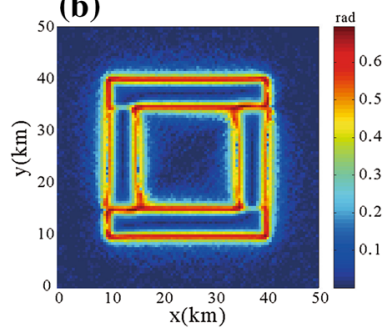

(e)

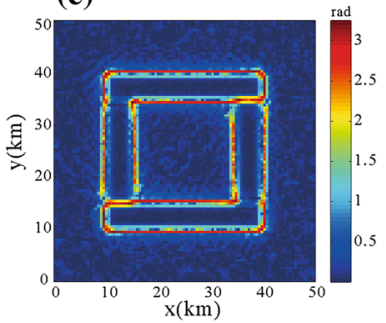

(c)

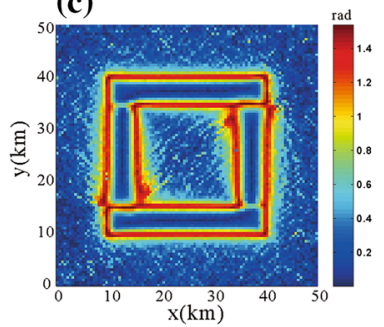

(f)

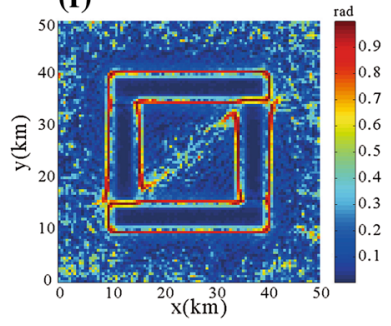

Fig. 8 Edges detected by new methods $N T$ and NFT with different $k$ values. a $N T$ of the data with $k$ value 0.2 . b $N T$ of the data with $k$ value 0.02 . $\mathbf{c} N T$ of the data with $k$ value 0.002 . d NFT of the data with $k$ value 0.2 . e $N F T$ of the data with $k$ value 0.02 . f $N F T$ of the data with $k$ value 0.002

It should be noted that when we use our new purposed edge detecting filters to interpret magnetic anomaly, the magnetic anomalies need to be reduced to the North Pole, as a fact that the magnetic anomaly derivatives are sensitive to the magnetization direction. 

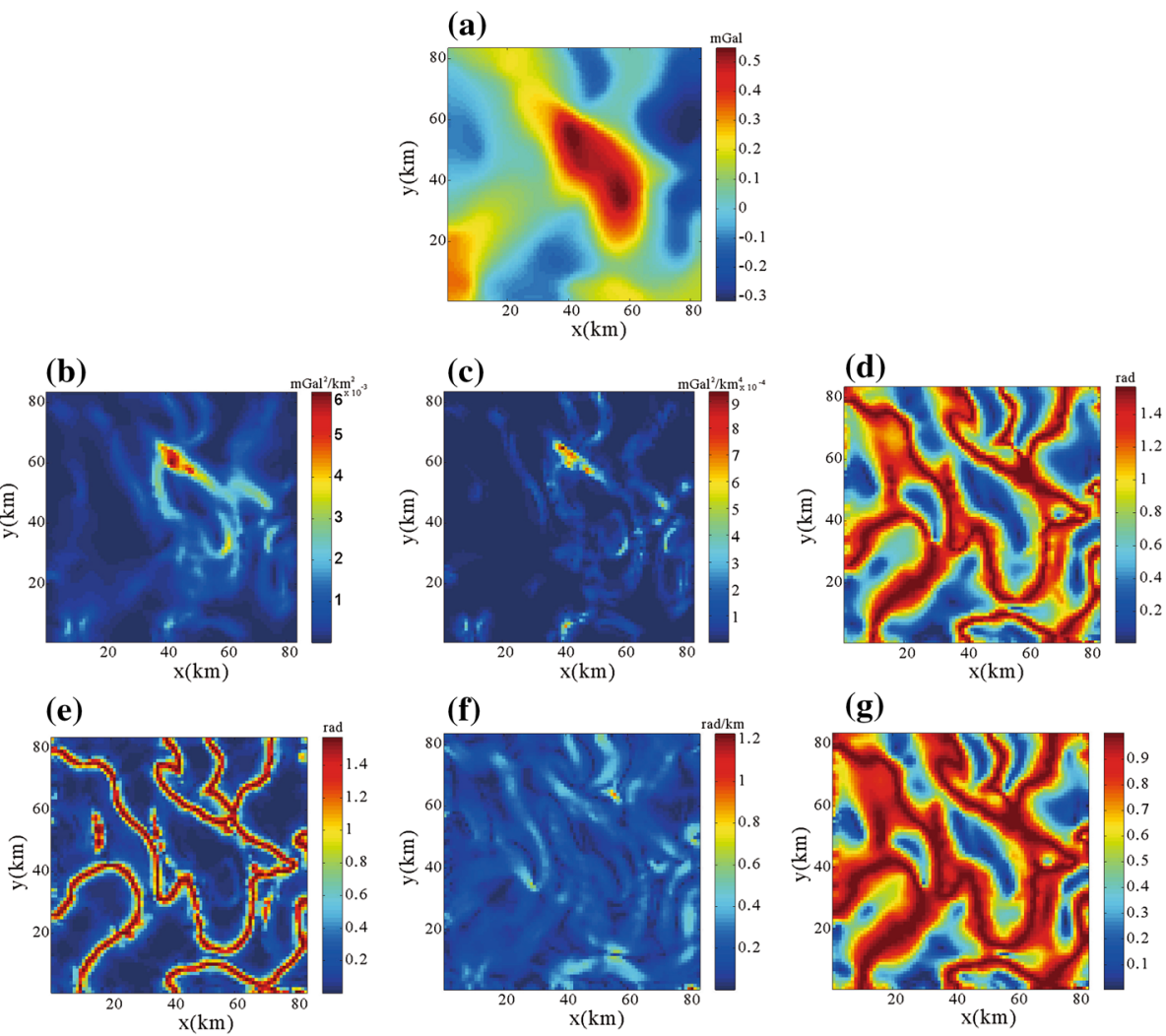

(g)

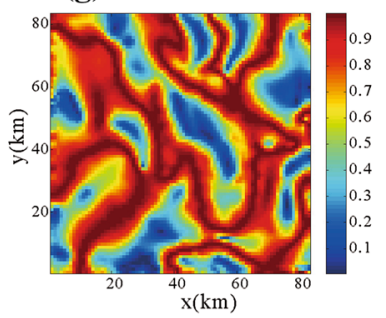

Fig. 9 Edges detected by different methods of gravity anomaly in Jilin province. a The gravity anomaly in Jilin province. b THDT of the data. c FTHDT of the data. d NT of the data. e NFT of the data. f TA_THDR of the data. g Theta map of the data

\section{Application to measured potential field data}

To test the application of the new purposed edge detector in real case, we apply the methods to measured gravity data. Figure 9a shows the residual gravity anomaly with $100 \mathrm{~m}$ sampling interval of a mine in Jilin province, Northern of China, which contains both positive and negative anomalies corresponding to different residual densities. The positive anomaly caused by magnetite and pyritization skarn in the center dominates the map. Figure $9 \mathrm{~b}-\mathrm{g}$ shows the different methods edge detecting results. It is hard to outline different amplitude anomalies for the new edge detectors THDT and FTHDT. The traditional edge detector TA_THDR in Fig. 9f can't outline the edges clearly with bad performance. And Theta map in Fig. 9g can recognize the edges, but they are diffused. The new normalized detectors $N T$ and $N F T$ can display the edges of different density contrast anomalies clearly and accurately. In this case, the value of $k$ is chosen as 0.0002 .

We also applied the new edge detectors to the measured magnetic anomalies in Zhurihe, Northeast, China. Figure 10a shows the magnetic anomalies data after the process of reduction to the pole. The study area size is $73 \times 117 \mathrm{~km}$ with $20 \mathrm{~m}$ sampling interval. The neoproterozoic supersequence consists predominantly of continental sediments and 

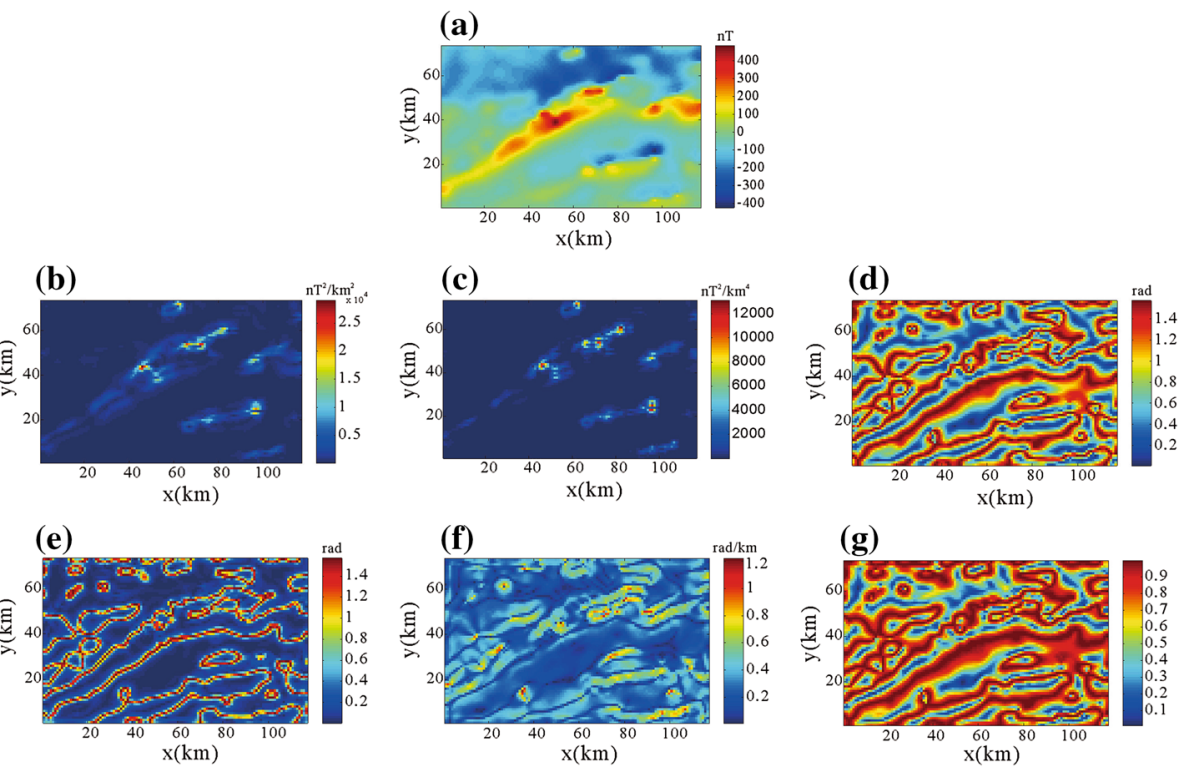

Fig. 10 Edges detected by different methods of magnetic anomaly in Zhurihe, China. a The gravity anomaly in Jilin province. b THDT of the data. c FTHDT of the data. d NT of the data. e NFT of the data. f TA_THDR of the data. $\mathbf{g}$ Theta map of the data

has reduced the intensity of the magnetic profiles, except for some iron-rich sandstone dykes. The nearly SE-NW trending dyes generate the nearly linear anomalies, and the iron structures dominate the map. The value of $\mathrm{k}$ is 0.0002 when using Eqs. (14) and (15) for calculating new normalized detectors $N T$ and NFT. The edge detecting performance of different methods is showed in Fig. 10b-g. NT and NFT have advantages in enhancing the small amplitude sources edges, especially the detector NFT. The NFT filter can not only outline the edges clearly and accurately, but also eliminate false edges for the complex geological situation compared with the traditional edge detectors.

\section{Conclusion}

In this paper, we present the edge detector based on directional total horizontal derivatives of three-dimensional structure tensor, and define two new edge detectors. We normalize the new detectors to outline strong and weak anomalies simultaneously using the differentorder vertical derivatives of potential field data. The normalized new edge detectors have the ability in eliminating false edges for the situation with positive and negative density contrast anomalies by introducing the parameter $k$ which should be determined by interpreter. The new normalized filters performance with different values of factor $\mathrm{k}$ is tested to explain the application effect of different parameters. Compared with other traditional methods on synthetic and real measured potential field data, our new purposed method can not only balance the different amplitude anomalies, but also has better resolution.

Acknowledgments We gratefully acknowledge the code shared by G. R. J. Cooper from University of the Witwatersrand, School of Geosciences, Johannesburg. This work was financially supported by SinoProbe09-01 (Grant No. 201311192). 


\section{References}

Beiki M (2010) Analytical signals of gravity gradient tensor and their application to estimate source location. Geophysics 75:I59-I74

Blakely RJ (1995) Potential theory in gravity and magnetic applications. Cambridge University Press, Cambridge

Cooper G (2009) Balancing images of potential-field data. Geophysics 74:L17-L20

Cooper G, Cowan D (2008) Edge enhancement of potential-field data using normalized statistics. Geophysics 73:H1-H4

Cordell L (1979) Gravimetric expression of graben faulting in Santa Fe Country and the Espanola Basin. In: 30th Field conference New Mexico. New Mexico Geological Society Guidebook, 59-64

Cordell L, Grauch VJS (1985) Mapping basement magnetization zones from aeromagnetic data in the San Juan basin, New Mexico. In: Hinze WJ (ed) The utility of regional gravity and magnetic anomaly society of exploration geophysics. Society of Exploration Geophysicists, New Orleans, pp 181-197

Hood PJ, Teskey DJ (1989) Aeromagnetic gradiometer program of the Geological Survey of Canada. Geophysics 54:1012-1022

Hsu SH, Sibuet JC, Shyu CT (1996) High-resolution detection of geologic boundaries from potential-field anomalies: an enhanced analytic signal technique. Geophysics 61(2):373-386

Jeong WK, Whitaker R, Dobin M (2006) Interactive 3D seismic fault detection on the Graphics Hardware. In proceedings of the International Workshop on Volume Graphics, 111-118

Ma GQ, Huang DN (2015) The removal of additional edges in the edge detection of potential field data. J Appl Geophys 114:168-173

Marson I, Klingele EE (2015) Advantages of using the vertical gradient of gravity for 3D interpretation. Geophysics 58(11):1588-1595

Miller HG, Singh V (1994) Potential field tilt-a new concept for location of potential field sources. J Appl Geophys 32:213-217

Nabighian MN (1972) The analytic signal of two-dimensional magnetic bodies with polygonal crosssection: its properties and use for automated anomaly interpretation. Geophysics 37:507-517

Nabighian MN (1984) Toward a three-dimensional automatic interpretation of potential field data via generalized Hilbert transforms: fundamental relations. Geophysics 49:780-786

Sertcelik I, Kafadar O (2012) Application of edge detection to potential field data using eigenvalue analysis of structure tensor. J Appl Geophys 84:86-94

Verduzco B, Fairhead JD, Green CM, MacKenzie C (2004) New insights into magnetic derivatives for structural mapping. Lead Edge 23:116-119

Wang HY, Ma, KK (2003) Automatic video object segmentation via 3D structure tensor. In: Proceedings. 2003 international conference on image processing I-153

Weickert J (1999a) Coherence-enhancing diffusion of color images. Image Vis Comput 17:199-210

Weickert J (1999b) Coherence-enhancing diffusion filtering. Int J Comput Vis 31:111-127

Wijns C, Perez C, Kowalczyk P (2005) Theta map: edge detection in magnetic data. Geophysics 70:L39_ L43

Yuan Y, Geng M (2014) Directional total horizontal derivatives of gravity gradient tensor and their application to delineat the edges. In: 76th EAGE conference \& exhibition, Amsterdam RAI, the Netherlands, 16-19 June 2014

Yuan Y, Huang DN, Yu QL, Lu PY (2014) Edge detection of potential field data with improved structure tensor methods. J Appl Geophys 108:35-42

Zhou WN, Du XJ, Li JY (2013) The limitation of curvature gravity gradient tensor for edge detection and a method for overcoming it. J Appl Geophys 98:237-242 